\title{
Treatment of chemotherapy induced anemia; a randomized clinical trial to compare quality of life in patients taking intravenous versus oral iron
}

\author{
Ali Hajigholami ${ }^{\circledR}$, Ahmad-Reza Maghsoodi $^{\circledR}{ }^{\circledR}$ Hourieh Ansari $^{3^{*}}{ }^{\circledR}$, Marzieh Kafeshani $^{4}$ \\ ${ }^{1}$ Department of Internal Medicine, Faculty of Medicine, Isfahan University of Medical Sciences, Isfahan, Iran \\ ${ }^{2}$ Department of Internal Medicine, Faculty of Medicine, Shahrekord University of Medical Sciences, Shahrekord, Iran \\ ${ }^{3}$ Family and Community Medicine Department, Faculty of Medicine, Isfahan University of Medical Sciences, Isfahan, Iran \\ ${ }^{4}$ School of Nutrition, Food Security Research Center, Isfahan University of Medical Sciences, Isfahan, Iran
}

*Correspondence to

Hourieh Ansari,

Houri_ansari@yahoo.com

Received 7 Aug. 2020 Accepted 23 Oct. 2020 Published online 14 Nov. 2020

Keywords: Chemotherapy, Erythropoietin, Hemoglobin, Iron, Quality of life

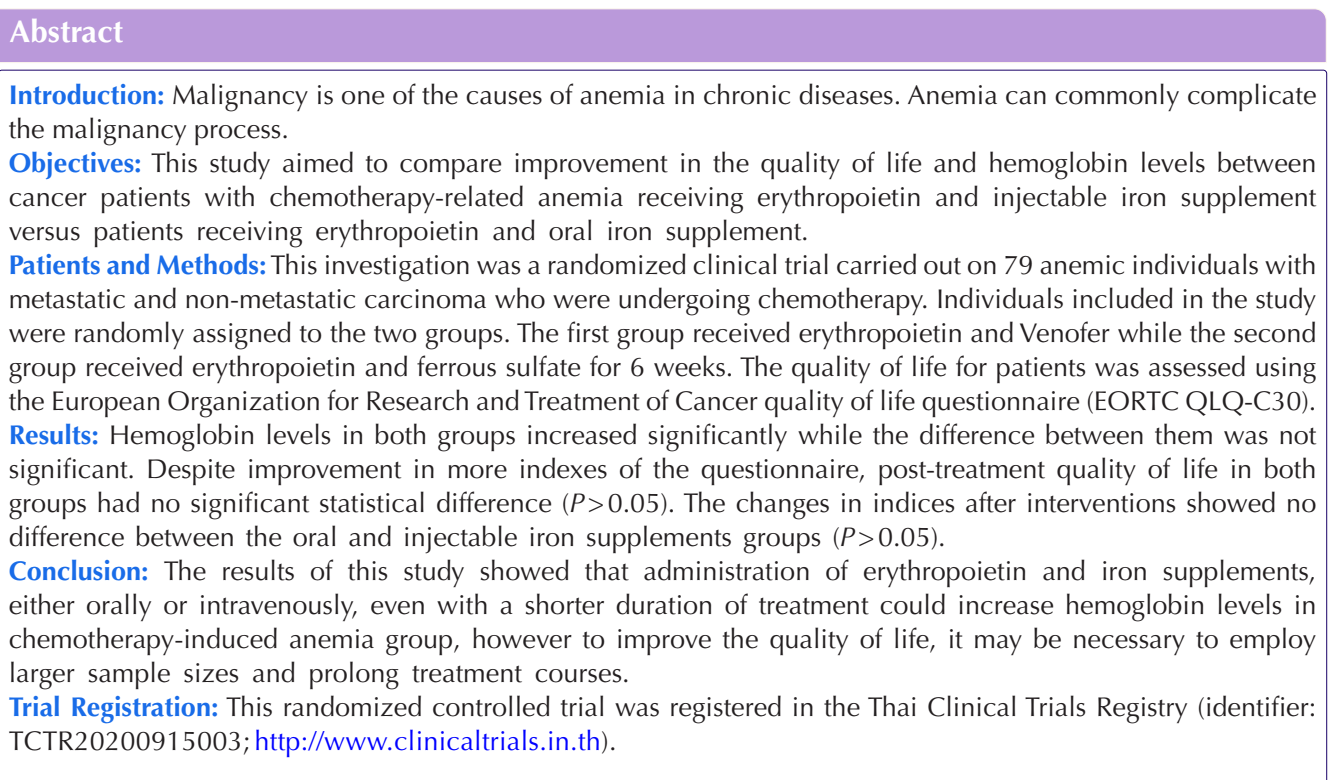

Citation: Hajigholami A, Maghsoodi AR, Ansari H, Kafeshani M. Treatment of chemotherapy induced anemia; a randomized clinical trial to compare quality of life in patients taking intravenous versus oral iron. Immunopathol Persa. $2021 ; 7(2): \mathrm{e} 32$. DOI:10.34172/ ipp.2021.32.

\section{Introduction}

Malignancy is one of the causes of anemia in chronic diseases (1). Anemia can commonly complicate the malignancy process which may be due to anti-neoplastic therapy (erythropoietin suppression through chemotherapy or radiation), progressive disease, the direct effect of malignancy (bleeding from tumors or bone marrow infiltration) and malignant products (amyloidinflammatory cytokines, auto-antibodies or microangiopathy) and also the effect of renal failure due to chemotherapy (2-4). Several factors involved in the development of anemia in the patients with malignancy such as abnormal metabolism of iron, or iron trapping in the macrophages, the inability to increase erythropoietin in response to anemia, a relative decrease in erythropoietin production, inflammation associated with the disease, myelosuppressive chemotherapy of malignancies, and other causes consisting of gastrointestinal bleeding, iron, folate and cobalamin deficiency (5-8). The management of anemia with chemotherapy, hematopoiesis stimulating agents such as erythropoietin or darbepoetin increase hemoglobin levels and reduce the need for blood transfusion. Regarding the lack of access to iron in the course of malignancy, the use of iron supplementation is necessary to develop a proper hematological response. Although some types of malignancies respond well to oral iron, it is still not proven that this treatment is effective enough in the patients receiving erythropoietin stimulating agents.

\footnotetext{
Copyright (C) 2021 The Author(s); Published by Nickan Research Institute. This is an open-access article distributed under the terms of the Creative Commons Attribution License (http://creativecommons.org/licenses/by/4.0), which permits unrestricted use, distribution, and reproduction in any medium, provided the original work is properly cited.
} 


\section{Key point}

In a randomized clinical trial carried out on 79 anemic individuals with metastatic and non-metastatic carcinoma who were undergoing chemotherapy, we found that the changes in indices after interventions showed no difference between the oral and injectable iron supplements groups. We concluded that administration of erythropoietin and iron supplements, both orally and intravenously, even with a shorter duration of treatment could increase hemoglobin levels in chemotherapy-induced anemia group.

However, injectable iron supplements can quickly amplify erythropoiesis by providing a bioavailable form of iron $(9,10)$. In addition, due to lower prices of oral drug, the use of oral iron compared with its injectable form reduces the cost of treatment for patients.

Malignant anemia can affect the patient's functional status, reduce the patient's physiological capacity as well as lead to fatigue and disability. Diagnosis and treatment of anemia can result in improved quality of life in patients with malignancy. In addition to the development of symptoms, the presence of anemia in some cancers worsens prognosis because the response to anticancer and ionizing radiation and some types of chemotherapy for cytotoxicity requires adequate oxygenation (11-14).

\section{Objectives}

In this study, we compared the quality of life, and hemoglobin levels between cancer patients with chemotherapy-related anemia receiving erythropoietin and injectable iron supplement with patients receiving erythropoietin and oral iron supplement.

\section{Patients and Methods}

\section{Study design}

This investigation was a randomized clinical trial conducted on 79 anemic individuals with metastatic and non-metastatic malignancy who were undergoing chemotherapy. The inclusion criteria were anemia secondary to malignancy and chemotherapy, the grading of anemia according to the National Cancer Institute (NCI) system( grade 0-4), remission phase in hematological malignancies and the Karnofsky performance status scale (an assessment tool for functional impairment) greater than or equal to $70 \%$. Exclusion criteria were unwillingness to continue cooperation during the study, banning from erythropoietin administration due to thromboembolic events or systolic blood pressure more than $160 \mathrm{~mm} \mathrm{Hg}$ during the study or any infection suffering from digestive disorders caused by oral iron intake or gastrointestinal bleeding, anemia due to other causes than malignancy, death due to complications of the illness, total gastrostomy, gastric cancer, lack of response to more than $20 \%$ of questions in the questionnaire or serum ferritin level greater than $500 \mathrm{ng} / \mathrm{mL}$. The patients who had inclusion criteria were randomly assigned to the two groups using a random number table and the European Organization for Research and Treatment of Cancer quality of life questionnaire (EORTC QLQ-C30) was completed for all patients (15) (Figure 1). The first group received erythropoietin (150 units/kg subcutaneously three times a week) and Venofer (100 mg, intravenously at each chemotherapy session), and the second group received erythropoietin (150 units/kg subcutaneously three times a week) and ferrous sulfate (one tablet every 8 hours) for six weeks. Hemoglobin less than $8 \mathrm{mg} / \mathrm{dL}$ or presence of organ dysfunction and coronary artery disease were considered as the limit of blood transfusion in patients. In order to reduce gastrointestinal complications and maximum drug intake by patients, the correct method of taking iron tablets was trained. In the oral iron receiving group, three patients were excluded from the study due to lack of completeness of the questionnaire, one patient due to oral iron intolerance and one person due to death during the treatment. In the group receiving the injectable iron, two patients were excluded from the study because of the lack of completion of the questionnaires, one patient due to lack of proper control of blood pressure and two persons due to lack of willingness to continue cooperation. Finally, 40 subjects in the oral group and 39 in the injectable group completed the study and at the end of the study, once again, the questionnaire was completed for both groups. In this study, blindness was not performed due to differences in iron administration in the two groups. Information about the patient's tests was extracted from the patient's file.

The quality of life was measured using EORTC (European Organization for Research and Treatment of Cancer quality of life questionnaire) QLQ-C30, a standard questionnaire for assessing the quality of life of cancer patients. The questionnaire consists of three parts, 1) General health status or quality of life, which includes two questions (Questions 29 and 30) with a scoring range from 1 (very bad) to 7 (excellent), 2) The functional status consists of five subsets; physical function includes five questions (Questions 1 to 5), social role including two questions (questions 6 and 7), emotional status including four questions (questions 21-24), cognitive status including two questions (question 20 and 25), social status includes two questions (questions 26 and 27), and 3) Symptoms include 9 items; fatigue including three questions (questions 10,12,18), nausea and vomiting including two questions (questions 14 and 15), pain including two questions (questions 9 and 19), shortness of breath including one question (question 8), sleeplessness includes a question (question 11), appetite reduction including one question (question 13), constipation including one question (question 16), diarrhea including one question (question 17), and financial problems including one question (question 28). The scoring for physical status and symptoms; was as not at all (1), low (2), high (3) and very high (4). The score for each item in the questionnaire ranged between 0 and 100 that the method of calculating score was based on the resource guide provided by the 


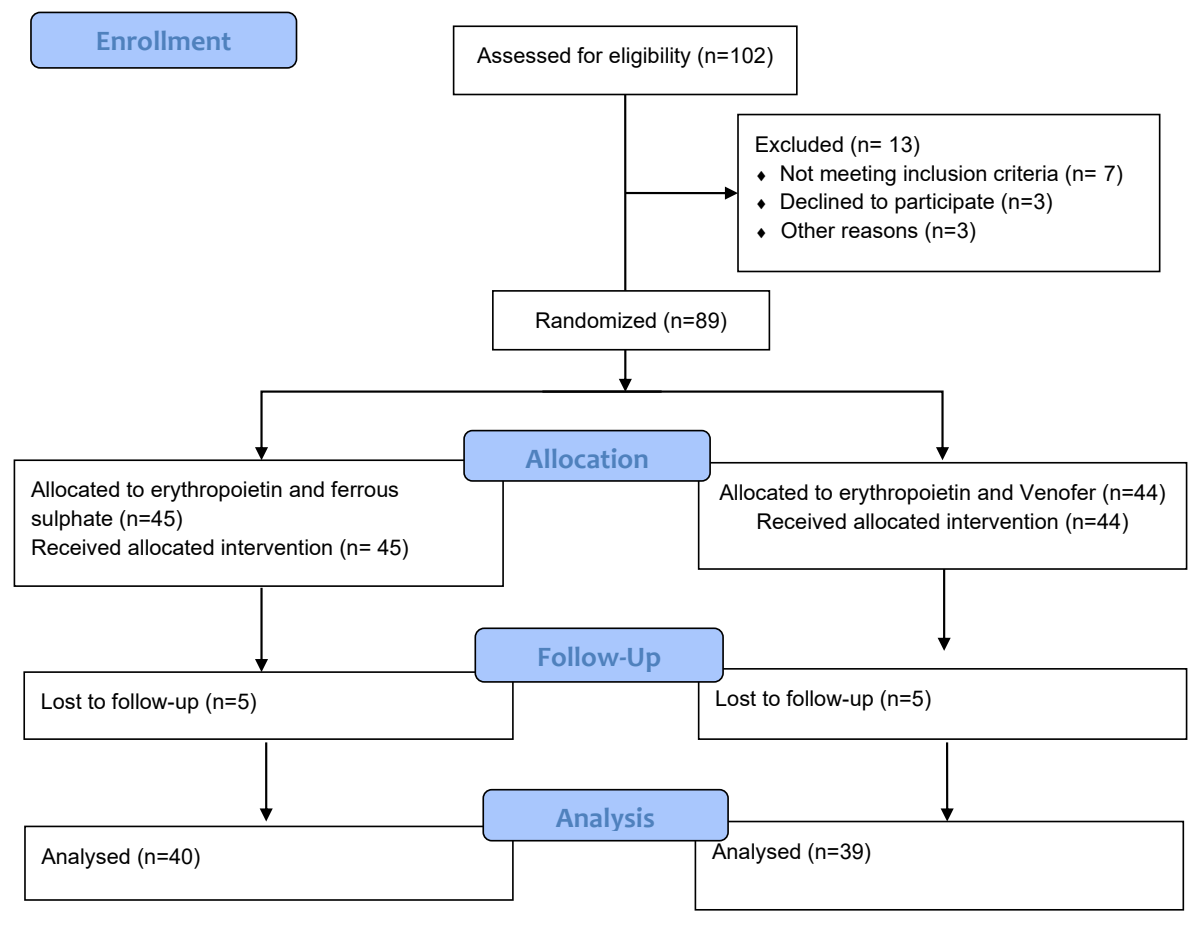

Figure 1. CONSORT flow diagram.

European Organization for Research and Treatment of Cancer Quality of Life (EORTC) as the statistical formulas for each group of different questions (15). A higher score in the functional section indicates higher levels of performance and a higher score in general health indicates a higher level of quality of life. The high score in symptoms suggests a high level of difficulty.

\section{Ethical issues}

The research followed the tenets of the Declaration of Helsinki. The study was approved by the institutional ethics committee of Shahrekord University of Medical Sciences and registered as a randomized clinical trial (identifier: TCTR20200915003; http://www.clinicaltrials. in.th/). Written informed consent was obtained from all participants too. This paper is extracted from the internal medicine residential thesis of Ahmad-Reza Maghsoudi (Thesis\# 997).

\section{Statistical analysis}

The SPSS version 18.0 (SPSS Inc., Chicago, IL) was applied for statistical analysis. The normality of data was checked using the Kolmogorov-Smirnov test. In this regard, age, hemoglobin level, quality of life score, physical status score, emotional status score and pain score had a normal distribution. For inter-group comparison, $t$ test was used for variables with normal distribution and Wilcoxon signed-rank test used for variables with abnormal distribution. Independent $t$ test and Mann-Whitney $\mathrm{U}$ test were used for variables with normal distribution and abnormal distribution respectively. Accordingly, $P$ values of 0.05 or less were considered significant.

\section{Results}

In the group receiving the oral iron supplement, the mean age was $48.1 \pm 14.6$ years ranging from 22 to 78 years, $55 \%$ were female and $82.5 \%$ were married. The mean serum level of hemoglobin before the intervention was $10.4 \pm 1.1$ $\mathrm{g} / \mathrm{dL}$ ranging from 8 to $12 \mathrm{~g} / \mathrm{dL}$ that reached $11.2 \pm 1.4 \mathrm{~g} / \mathrm{dL}$ after intervention with a significant increase $(P=0.005)$. In the group receiving the injectable iron supplement, the mean age was $50.9 \pm 13.1$ years ranging from 20 to 84 years. The mean serum hemoglobin level before the intervention was $10.1 \pm 1.3 \mathrm{~g} / \mathrm{dL}$ ranging from 7 to $12 \mathrm{~g} / \mathrm{dL}$ and after the intervention was $11.4 \pm 1.6 \mathrm{~g} / \mathrm{dL}$ that indicates significant change after drug administration $(P<0.001$; Table 1$)$.

In the patients who received injectable iron, the level of quality of life, physical function score, and emotional score, the scores for fatigue, pain, insomnia, constipation nausea, anorexia, and diarrhea did not change significantly (Table 2 ), however the physical index increased after intervention compared to the previous status $(P=0.005)$. In the group received oral iron, all indices did not change significantly after intervention (Table 3), nevertheless similar to another

Table 1. Baseline characteristics of the patient participated in the study

\begin{tabular}{llccc}
\hline Variable & & $\begin{array}{c}\text { Group I } \\
(\mathbf{n}=\mathbf{3 9})\end{array}$ & $\begin{array}{c}\text { Group II } \\
(\mathbf{n}=\mathbf{4 0})\end{array}$ & P value \\
\hline Age $(\mathrm{y})$ & & $50.90 \pm 13.1$ & $41.8 \pm 16.6$ & 0.37 \\
\multirow{2}{*}{ Gender, No (\%) } & Female & $23(59)$ & $22(55)$ & 0.72 \\
& Male & $16(41)$ & $18(45)$ & \\
\hline
\end{tabular}


group, the physical index increased after intervention $(P=0.02)$. Additionally, the changes in indices after interventions showed no difference between the oral and injectable iron supplements groups $(P>0.05$; Table 4$)$. The change in serum hemoglobin level between the two groups showed no significant difference $(P=0.665)$.

\section{Discussion}

In our study, the serum hemoglobin level increased in both groups however, this increase had no significant difference between groups. These results disagree with the findings of other studies, such as Henry et al (16) that revealed the use of injectable iron supplement caused more improvement in serum hemoglobin level compared to oral iron supplement. The possible reason for this significant change in the study by Henry et al could be related to weekly administrating injectable iron or considering an 8-week course for treatment, since in our study, the patients received intravenous iron every three weeks and the duration of the treatment was six weeks (totally, the patients received intravenous iron twice in six weeks).

Another possible explanation for the lack of a significant difference in increased hemoglobin during injectable versus oral iron group may be due to the effect of erythropoietin too.

Straus et al (14) showed that the rapid treatment of mild anemia with erythropoietin in the patients with hematological malignancies especially lymphoma significantly improved the quality of life. A review study by Katodritou et al (17) indicated that co-administration of erythropoietin and iron supplement intravenously improved hematological response. Our study was

Table 2. Changes in the mean quality of life scores in the group received erythropoietin and ferrous sulfate

\begin{tabular}{lccc}
\hline Variable & Baseline & $\mathbf{6}$ weeks & $\boldsymbol{P}$ value \\
\hline Global health status & $56.25 \pm 20.47$ & $57.50 \pm 3.25$ & 0.74 \\
Functional status & & & \\
Physical function & $64.16 \pm 17.84$ & $74 \pm 21.44$ & 0.02 \\
Social function & $69.16 \pm 5.31$ & $74.16 \pm 32.89$ & 0.39 \\
Emotional function & $58.95 \pm 0.85$ & $66.66 \pm 27.86$ & 0.22 \\
Cognitive function & $88.75 \pm 6.61$ & $81.25 \pm 4.22$ & 0.08 \\
Functional function & $79.16 \pm 3.79$ & $78.33 \pm 9.28$ & 0.79 \\
Hemoglobin (g/dl) & $10.4 \pm 1.1$ & $11.2 \pm 1.4$ & $<0.01$ \\
Symptoms & & & \\
Shortness of breath & $9.16 \pm 21.33$ & $15.83 \pm 31.11$ & 0.29 \\
Pain & $35.83 \pm 29.36$ & $32.08 \pm 31.66$ & 0.49 \\
Sleeplessness & $50 \pm 35.40$ & $43.33 \pm 38.63$ & 0.33 \\
Appetite reduction & $30.83 \pm 34.90$ & $31.66 \pm 4.55$ & 0.89 \\
Constipation & $25 \pm 33.54$ & $6.66 \pm 34.08$ & 0.90 \\
Diarrhea & $5 \pm 14.22$ & $6.66 \pm 17.21$ & 0.53 \\
Financial problems & $55 \pm 40.33$ & $51.66 \pm 6.50$ & 0.55 \\
Nausea & $15.41 \pm 21.14$ & $20.83 \pm 28.68$ & 0.14 \\
Fatigue & $48.61 \pm 28.76$ & $40 \pm 29.28$ & 0.16 \\
\hline & & & \\
\hline
\end{tabular}

conducted on patients with severe anemia; hence it takes more time to regain an acceptable level of quality of life. In the study by Lind et al (12), a longer course of treatment led to a normalization of hemoglobin levels whereas, in our study, the mean of hemoglobin level had not reached normal levels in any of the two groups. Perhaps due to this reason, despite a significant increase in hemoglobin levels in both groups, improvement in the quality of life has

Table 3. Changes in the mean quality of life scores in the group received erythropoietin and Venofer

\begin{tabular}{lccc}
\hline Variable & Baseline & $\mathbf{6}$ weeks & $\boldsymbol{P}$ value \\
\hline Global health status & $52.13 \pm 27.61$ & $54.27 \pm 23.17$ & 0.68 \\
Functional status & & & \\
Physical function & $62.05 \pm 23.55$ & $72.47 \pm 23.20$ & $<0.001$ \\
Social function & $61.96 \pm 31.28$ & $71.36 \pm 27.55$ & 0.09 \\
Emotional function & $58.76 \pm 25.85$ & $65.59 \pm 26.29$ & 0.22 \\
Cognitive function & $77.35 \pm 21.41$ & $75.64 \pm 23.20$ & 0.78 \\
Functional function & $69.23 \pm 27.71$ & $69.2 \pm 27.71$ & 0.79 \\
Hemoglobin (g/dL) & $10.1 \pm 1.30$ & $11.4 \pm 1.6$ & $<0.001$ \\
Symptoms & & & \\
\hline Shortness of breath & $16.23 \pm 24.02$ & $13.67 \pm 18.28$ & 0.29 \\
Pain & $47.43 \pm 27.44$ & $40.59 \pm 29.06$ & 0.27 \\
Sleeplessness & $41.88 \pm 34.80$ & $37.04 \pm 30.53$ & 0.27 \\
Appetite reduction & $41.02 \pm 32.85$ & $34.18 \pm 28.08$ & 0.30 \\
Constipation & $29.05 \pm 34.35$ & $25.6 \pm 34.08$ & 0.70 \\
Diarrhea & $13.67 \pm 19.82$ & $17.94 \pm 26.32$ & 0.32 \\
Financial problems & $52.99 \pm 37.24$ & $51.28 \pm 38.11$ & 0.83 \\
Nausea & $27.35 \pm 29.44$ & $22.64 \pm 18.92$ & 0.14 \\
Fatigue & $48.14 \pm 27.96$ & $44.15 \pm 24.11$ & 0.16 \\
\hline
\end{tabular}

Table 4. Changes in the mean quality of life scores in the group received erythropoietin and Venofer with group received erythropoietin and ferrous sulfate after intervention

\begin{tabular}{lccc}
\hline Variable & Baseline & $\mathbf{6}$ weeks & $\boldsymbol{P}$ value \\
\hline Global health status & $54.27 \pm 23.17$ & $57.50 \pm 3.25$ & 0.71 \\
Functional status & & & \\
Physical function & $72.47 \pm 23.20$ & $74 \pm 21.44$ & 0.99 \\
Social function & $71.36 \pm 27.55$ & $74.16 \pm 32.89$ & 0.25 \\
Emotional function & $65.59 \pm 26.29$ & $66.66 \pm 27.86$ & 0.86 \\
Cognitive function & $75.64 \pm 23.20$ & $81.25 \pm 4.22$ & 0.20 \\
Functional function & $69.2 \pm 27.71$ & $78.33 \pm 9.28$ & 0.08 \\
Hemoglobin (g/dl) & $11.4 \pm 1.6$ & $11.2 \pm 1.4$ & 0.66 \\
Symptoms & & & \\
\hline Shortness of breath & $13.67 \pm 18.28$ & $15.83 \pm 31.11$ & 0.48 \\
Pain & $40.59 \pm 29.06$ & $32.08 \pm 31.66$ & 0.15 \\
Sleeplessness & $37.04 \pm 30.53$ & $43.33 \pm 38.63$ & 0.39 \\
Appetite reduction & $34.18 \pm 28.08$ & $31.66 \pm 4.55$ & 0.41 \\
Constipation & $25.6 \pm 34.08$ & $6.66 \pm 34.08$ & 0.27 \\
Diarrhea & $17.94 \pm 26.32$ & $6.66 \pm 17.21$ & 0.02 \\
Financial problems & $51.28 \pm 38.11$ & $51.66 \pm 6.50$ & 0.99 \\
Nausea & $15.41 \pm 21.14$ & $20.83 \pm 28.68$ & 0.16 \\
Fatigue & $48.61 \pm 28.76$ & $40 \pm 29.28$ & 0.32 \\
\hline
\end{tabular}


not been observed. Accordingly, we found the beneficial effects of erythropoietin to improve brain ischemia. Hence, administration of erythropoietin in cancer patients had various ameliorative effects beyond treatment of anemia $(18-20)$.

\section{Conclusion}

The results of this study showed that administration of erythropoietin and iron supplements, both orally and intravenously, even with a shorter duration of treatment (as compared to other studies) could increase hemoglobin levels in chemotherapy-induced anemia group, but in order to improve the quality of life, it may be necessary to employ larger sample sizes and prolong treatment courses.

\section{Limitations of the study}

Given the contradicting results of this and other studies, larger-scale studies are recommended to be conducted on the long-term effects of contrast agents on renal function.

\section{Authors' contribution}

AHG and ARM conducted the research and contributed to the conception and design of the research. HA and MK prepared the primary draft. ARM contributed to the acquisition of data. AHG finalized the paper. All authors read and signed the final paper.

\section{Conflicts of interest}

The authors declare that they have no competing interests.

\section{Ethical consideration}

Ethical issues (including plagiarism, data fabrication, double publication) have been completely observed by the authors

\section{Funding/Support}

This study was supported by Shahrekord University of Medical Sciences, Shahrekord, Iran.

\section{References}

1. Ludwig H, Müldür E, Endler G, Hübl W. Prevalence of iron deficiency across different tumors and its association with poor performance status, disease status and anemia. Ann Oncol. 2013;24:1886-92. doi: 10.1093/annonc/mdt118.

2. Williams LA, Bohac C, Hunter S, Cella D. Patient and health care provider perceptions of cancer-related fatigue and pain. Support Care Cancer. 2016;24:4357-63. doi: 10.1007/s00520016-3275-2

3. Littlewood T, Bajetta E, Nortier J, Vercammen E, Rapoport B, Group EAS. Effects of epoetin alfa on hematologic parameters and quality of life in cancer patients receiving nonplatinum chemotherapy: results of a randomized, double-blind, placebocontrolled trial. J Clin Oncol. 2001;19:2865-74. doi: 10.1200/ JCO.2001.19.11.2865.

4. Gabrilove JL, Cleeland CS, Livingston RB, Sarokhan B, Winer $\mathrm{E}$, Einhorn LH. Clinical evaluation of once-weekly dosing of epoetin alfa in chemotherapy patients: improvements in hemoglobin and quality of life are similar to three-timesweekly dosing. J Clin Oncol. 2001;19:2875-82. doi: 10.1200/ JCO.2001.19.11.2875.

5. Ludwiczek S, Aigner E, Theurl I, Weiss G. Cytokine-mediated regulation of iron transport in human monocytic cells. Blood.
2003;101:4148-54 doi: 10.1182/blood-2002-08-2459.

6. Theurl I, Mattle V, Seifert M, Mariani M, Marth C, Weiss G. Dysregulated monocyte iron homeostasis and erythropoietin formation in patients with anemia of chronic disease. Blood. 2006;107:4142-8. doi: 10.1182/blood-2005-08-3364.

7. Papadaki HA, Kritikos HD, Valatas V, Boumpas DT, Eliopoulos GD. Anemia of chronic disease in rheumatoid arthritis is associated with increased apoptosis of bone marrow erythroid cells: improvement following anti-tumor necrosis factor- $\alpha$ antibody therapy. Blood. 2002;100:474-82. doi: 10.1182/ blood-2002-01-0136.

8. Weiss G, Goodnough LT. Anemia of chronic disease. N Engl Med. 2005;352:1011-23. doi: 10.1056/NEJMra041809.

9. Rizzo JD, Brouwers M, Hurley P, Seidenfeld J, Arcasoy MO, Spivak $J \mathrm{~L}$, et al. American Society of Hematology/American Society of Clinical Oncology clinical practice guideline update on the use of epoetin and darbepoetin in adult patients with cancer. Blood. 2010:116:4045-59. doi: 10.1182/blood-2010-08-300541.

10. Rizzo JD, Brouwers M, Hurley P, Seidenfeld J, Arcasoy MO, Spivak JL, et al. American Society of Clinical Oncology/ American Society of Hematology clinical practice guideline update on the use of epoetin and darbepoetin in adult patients with cancer. J Clin Oncol. 2010;28:4996-5010. doi: 10.1200/ JCO.2010.29.2201

11. Cash JM, Sears DA. The anemia of chronic disease: spectrum of associated diseases in a series of unselected hospitalized patients. Am J Med. 1989;87:638-44. doi: 10.1016/s00029343(89)80396-1.

12. Lind $M$, Vernon $C$, Cruickshank D, Wilkinson $P$, Littlewood $T$, Stuart $\mathrm{N}$, et al. The level of haemoglobin in anaemic cancer patients correlates positively with quality of life. $\mathrm{Br} J$ Cancer. 2002;86:1243-9. doi: 10.1038/sj.bjc.6600247.

13. Samol J, Littlewood TJ. The Efficacy of rHuEPO in Cancer-Related Anaemia. Br J Hematol. 2003;121:3-11. doi: 10.1046/j.13652141.2003.04129.x.

14. Straus DJ, Testa MA, Sarokhan BJ, Czuczman MS, Tulpule A, Turner RR, et al. Quality-of-life and health benefits of early treatment of mild anemia. Cancer. 2006;107:1909-17. doi: 10.1002/cncr.22221.

15. Fayers P, Bottomley A, Group EQoL. Quality of life research within the EORTC - the EORTC QLQ-C30. Eur J Cancer. 2002;38:125-33. doi: 10.1016/s0959-8049(01)00448-8.

16. Henry DH, Dahl NV, Auerbach M, Tchekmedyian S, Laufman LR. Intravenous ferric gluconate significantly improves response to epoetin alfa versus oral iron or no iron in anemic patients with cancer receiving chemotherapy. Oncologist. 2007;12:231 42. doi: 10.1634/theoncologist.12-2-231.

17. Katodritou E, Zervas K, Terpos E, Brugnara C. Use of erythropoiesis stimulating agents and intravenous iron for cancer and treatment-related anaemia: the need for predictors and indicators of effectiveness has not abated. $\mathrm{Br} J$ Hematol. 2008;142:3-10. doi: 10.1111/j.1365-2141.2008.07163.x.

18. Juul SE, Comstock BA, Heagerty PJ, Mayock DE, Goodman AM, Hauge S, Gonzalez F, Wu YW. High-Dose Erythropoietin for Asphyxia and Encephalopathy (HEAL): A Randomized Controlled Trial - Background, Aims, and Study Protocol. Neonatology. 2018;113:331-8. doi: 10.1159/000486820.

19. Hernández CC, Burgos CF, Gajardo AH, Silva-Grecchi T, Gavilan J, Toledo JR, et al. Neuroprotective effects of erythropoietin on neurodegenerative and ischemic brain diseases: the role of erythropoietin receptor. Neural Regen Res. 2017;12:1381-9. doi: 10.4103/1673-5374.215240.

20. Sgrò P, Sansone M, Sansone A, Romanelli F, Di Luigi L. Effects of erythropoietin abuse on exercise performance. Phys Sportsmed. 2018;46(1):105-15. doi: 10.1080/00913847.2018.1402663. 